\title{
Mechanismen-orientierte Schmerztherapie Neuropathische Schmerzen benötigen eine spezielle Therapie
}

- Verschiedene Schmerzzustände können sowohl nozizeptive als auch neuropathische Komponenten aufweisen. Neuropathische Schmerzen sollten differenzialdiagnostisch von nozizeptiven abgegrenzt und entsprechend behandelt werden.

Neuropathischen Schmerzen liegt eine Läsion von Nervenstrukturen im peripheren und/oder zentralen Nervensystem zugrunde. Der Therapieentscheidung müsse eine suffiziente Schmerzanalyse vorausgehen, berichtete Professor Thomas Tölle, Klinikum rechts der Isar der Technischen Universität München. Für die klinische Routine eigne sich eine Reihe einfacher Untersuchungsverfahren wie die Bestimmung der Schmerz-, Berührungs-, Druck-, Temperatur- und Vibrationsempfindung und der Muskeleigenreflexe. Die „Quantitative Sensorische Testung“(QST) ermögliche darüber hinaus auch die Diagnostik einer Small-Fiber-Neuropathie. Ein effektives und einfach anzuwendendes Instrument zur differenzierten Darstellung neuropathischer und nozizeptiver Schmerzkomponenten sei der painDetect ${ }^{\circ}$ Fragebogen.

Ein Wirkstoff, der sich bei peripheren und zentralen neuropathischen Schmerzen bewährt hat, ist Pregabalin $\left(\right.$ Lyrica $\left.^{\circledR}\right)$. Die Substanz ist dem chemisch verwandten
Gabapentin in Bezug auf den Wirkungseintritt und die Wirkstärke überlegen, wie PD Dr. Michael Überall, Nürnberg, anhand der Daten einer prospektiven, nicht interventionellen Studie verdeutlichte. Insgesamt 305 Patienten wurden über bis zu drei Monate mit Pregabalin oder Gabapentin behandelt. Nach acht Wochen war ein Cross-Over möglich. Beide Substanzen führten laut Überall zwar zu einer Reduktion der neuropathischen Schmerzen; Pregabalin war aber in Bezug auf den Wirkeintritt und das Ausmaß der Beschwerdelinderung deutlich überlegen. In den ersten zwei Wochen waren die Unterschiede zwischen den beiden Substanzen am größten. Nach 14 Tagen erreichten unter Pregabalin rund $40 \%$ der Patienten ihr individuelles Behandlungsziel gegenüber $22 \%$ unter Gabapentin. Von einer Umstellung auf die jeweils andere Substanz in Woche 8 profitierten lediglich die Patienten, die zuvor mit Gabapentin behandelt wurden. Bei Patienten, die von Pregabalin auf Gabapentin wechselten, kam es zu einem Verlust an analgetischer Wirkung. Abdol A. Ameri

Symposium „Was wann bzw. wann was besser nicht? Neuropathische Schmerzen“ im Rahmen des Deutschen Schmerz- und Palliativtages, Frankfurt, 17.3.2012 Veranstalter Pfizer

\section{Multiple Sklerose}

\section{Patientenfreundliche Anwendung verbessert den Therapieerfolg}

- Das Basistherapeutikum Interferon(IFN) beta-1a (Avonex ${ }^{\circledast}$ ) bietet Patienten mit schubförmiger Multipler Sklerose (RRMS) auch langfristig eine wirksame und sichere Behandlungsoption, die darüber hinaus hautverträglich und unkompliziert in der Anwendung ist.

PD Dr. med. Ralf Linker, Universitätsklinikum Erlangen, hob die Bedeutung der Adhärenz für den Therapieerfolg hervor. In puncto Wirksamkeit und Sicherheit seien gängige Basistherapeutika vergleichbar, Unterschiede gebe es vor allem hinsichtlich der Applikationsfrequenz. „Je seltener die Patienten spritzen müssen, desto zuverlässiger halten sie sich an die Therapiervorgaben“, betonte er. So konnten unter der nur einmal wöchentlichen Behandlung mit IFN beta-1a i. m. im Vergleich zu anderen Basistherapeutika signifikant höhere Adhärenzraten von $85 \%$ erzielt werden. Dies wiederum wirke sich positiv auf die Krankheitsaktivität und die Lebensqualität der Patienten aus.

Linker ist überzeugt, dass sich die Therapietreue mit dem im Juni 2011 eingeführten neuen Autoinjektor (Avonex ${ }^{\circledast}$ Pen $^{\mathrm{TM}}$ ) dank einfacher Handhabung und kaum spürbarer Injektion weiter verbessern wird. In der $\mathrm{Zu}$ lassungsstudie hatten $94 \%$ der Patienten den WochenPen der Fertigspritze vorgezogen.
Als weiteren Pluspunkt nannte der Neuroimmunologe die gute Hautverträglichkeit des Präparates. „Nach intramuskulärer Injektion von IFN beta-1a treten deutlich weniger Lokalreaktionen auf als bei Mitteln, die in das immunologisch aktive Fettgewebe gespritzt werden“, erklärte er. Irreversible Gewebsschädigungen wie Narben oder Lipoatrophie seien für viele Patienten mit Leidensdruck und Stigmatisierung assoziiert.

Dass MS-Patienten auch nachhaltig von einer frühen immunmodulatorischen Basistherapie mit IFN-beta-1a profitieren, hätten aktuelle Zehn-Jahresdaten gezeigt [Kinkel RP et al. Arch Neurol 2012,69(2):183-190]. Die Tatsache, dass $92 \%$ der Patienten nach dieser langen Zeit noch einen schubförmigen MS-Verlauf aufweisen, sieht Linker als „erfreulichste Nachricht“. „Diese Ergebnisse sprechen dafür, dass wir über eine wirksame Entzündungsmodulation letztlich auch einen neuroprotektiven Effekt erreichen“, folgerte er.

Dr. Martina-Jasmin Utzt

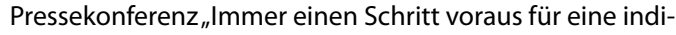
vidualisierte Therapie: Innovative Behandlungsstrategien", München, 29. Februar 2012; Biogen Idec 